\title{
Aplicação de um método de múltiplo estágio ao estudo da equação de transporte de soluto $2 \mathrm{D}$
}

\author{
Junior Francisco Dias, Neyva Maria Lopes Romeiro, \\ Depto de Matemática, CCE, UEL, \\ 86051-980, Londrina, PR \\ E-mail: juniordiasjfd@gmail.com, nromeiro@uel.br
}

Resumo: Em dinâmica de fluidos computacional há o interesse em se obter aproximações numéricas para equações diferenciais parciais. Neste trabalho o objetivo é discutir um dos métodos que pode ser utilizado para obter aproximações para a solução da equação de transporte bidimensional (2D). Para tanto é utilizado o aproximante de Padé $R_{2,2}$ para obter soluções por meio de um método de múltiplo estágio, conhecido como método de Harten/Tal-Ezer, atribuído a Amiram Harten (1946-1994) e Hillel Tal-Ezer. Após a apresentação de cada método são feitas simulações computacionais nas quais pode-se compará-los. A partir destas simulações conclue-se que o método de Harten/Tal-Ezer é mais vantajoso que os demais.

Palavras-chave: Equação de transporte, Método de múltiplo estágio (Harten/Tal-Ezer), Aproximantes de Padé, Método de Crank-Nicolson

\section{Introdução}

Os perigos veiculados pela água são muitos e estão presentes na vida de todos. Segundo [3], a poluição e contaminação desse recurso mata mais do que todas as formas de violência juntas, incluindo as guerras. No mundo, o índice de mortalidade infantil decorrente de doenças causadas pela má qualidade da água é de $17 \%$. Além disso, atualmente, mais da metade dos leitos hospitalares são ocupados por pessoas com doenças relacionadas a água contaminada.

Apesar de se conhecer a necessidade do monitoramento constante da concentração de poluentes na água, nem sempre esse controle ocorre satisfatoriamente. Como os poluentes não ficam distribuídos de maneira homogênea em um curso d'água, não basta coletar amostras em um só lugar para inferir sua qualidade e saber se esta é própria para consumo. Além disso, dependendo de quais substâncias estão presentes, a água deve receber tratamento diferenciado. Com um número alto de amostras a serem analisadas, as quais devem ser coletadas em diversos pontos, a realização manual desse trabalho se torna lenta e cara. Por esses e outros motivos, uma das estratégias que vem sendo largamente discutida no meio acadêmico é a utilização de métodos computacionais, principalmente no que diz respeito à equação de transporte.

\section{Equação governante}

Uma das maneiras de estudar a concentração de determinado poluente em um meio aquático é utilizar a equação de transporte. Ela é deduzida utilizando os mesmos princípios físicos que as equações de Navier-Stokes. Nesse trabalho discute-se o modelo de adveção-dispersão-reação bidimensional (2D), o qual pode ser encontrado em [2]. Em geral, essa versão da equação pode ser utilizada quando a profundidade do canal é bem menor que sua largura. Nesse caso, a simplificação para a versão 2D gera bons resultados. A equação em questão é dada por 


$$
\frac{\partial C}{\partial t}=D_{x x} \frac{\partial^{2} C}{\partial x^{2}}+D_{y y} \frac{\partial^{2} C}{\partial y^{2}}-v_{x} \frac{\partial C}{\partial x}-v_{y} \frac{\partial C}{\partial y}-k C,
$$

onde $C$ é a concentração dissolvida, $k$ é o coeficiente de primeira ordem da taxa de reação, $D_{x x}$ e $D_{y y}$ são os coeficientes dos termos de dispersão e $v_{x}$ e $v_{y}$ são as velocidades de fluxo.

\section{Os aproximantes de Padé}

As funções denominadas aproximantes de Padé são generalizações da série de Taylor. Para mais detalhes ver [1]. Assim como é possível expandir uma função em série de Taylor, pode-se expandí-la também utilizando os aproximantes de Padé.

Seguindo o raciocício proposto em [4], suponha que $C^{n+1}$ seja uma solução no nível de tempo $n+1$ para a equação de transporte, a qual pode-se supor que possa ser aproximada pela expansão de Taylor a partir de $C^{n}$, uma solução no nível de tempo $n$. Assim, tem-se

$$
C^{n+1}=C^{n}+\Delta t \frac{\partial}{\partial t} C^{n}+\frac{1}{2 !} \Delta t^{2} \frac{\partial^{2}}{\partial t^{2}} C^{n}+\ldots=\left(1+\Delta t \frac{\partial}{\partial t}+\frac{1}{2 !} \Delta t^{2} \frac{\partial^{2}}{\partial t^{2}}+\ldots\right) C^{n} .
$$

Portanto, $C^{n+1}$ pode ser representada como $\exp \left(\Delta t \frac{\partial}{\partial t}\right) C^{n}$. Utilizando os aproximantes de Padé pode-se representar $\exp (z)$ por uma função racional. O aproximante utilizado é conhecido como $R_{2,2}$ e é dado por

$$
\exp (z) \simeq R_{2,2}(z)=\frac{1+\frac{1}{2} z+\frac{1}{12} z^{2}}{1-\frac{1}{2} z+\frac{1}{12} z^{2}}
$$

A variável $z$ pode ser substituída por $\Delta t \frac{\partial}{\partial t}$ na equação (3), a qual pode ser reescrita como

$$
\exp \left(\Delta t \frac{\partial}{\partial t}\right) \simeq \frac{1+\frac{1}{2} \Delta t \frac{\partial}{\partial t}+\frac{1}{12}\left(\Delta t \frac{\partial}{\partial t}\right)^{2}}{1-\frac{1}{2} \Delta t \frac{\partial}{\partial t}+\frac{1}{12}\left(\Delta t \frac{\partial}{\partial t}\right)^{2}}
$$

Substituindo (4) em (2), tem-se

$$
\begin{aligned}
& {\left[1-\frac{1}{2} \Delta t \frac{\partial}{\partial t}+\frac{1}{12}\left(\Delta t \frac{\partial}{\partial t}\right)^{2}\right] C^{n+1}=\left[1+\frac{1}{2} \Delta t \frac{\partial}{\partial t}+\frac{1}{12}\left(\Delta t \frac{\partial}{\partial t}\right)^{2}\right] C^{n} \Leftrightarrow } \\
\Leftrightarrow & {\left[1-\frac{1}{2} \Delta t \frac{\partial}{\partial t}\left(1-\frac{1}{6} \Delta t \frac{\partial}{\partial t}\right)\right] C^{n+1}=\left[1+\frac{1}{2} \Delta t \frac{\partial}{\partial t}\left(1+\frac{1}{6} \Delta t \frac{\partial}{\partial t}\right)\right] C^{n} . }
\end{aligned}
$$

De acordo com a série de Taylor,

$$
\begin{gathered}
C^{n+5 / 6}=\frac{C^{n+1}}{0 !}+\left(-\frac{\Delta t}{6}\right) \frac{\partial}{\partial t} \frac{C^{n+1}}{1 !}+\left(-\frac{\Delta t}{6}\right)^{2} \frac{\partial^{2}}{\partial t^{2}} \frac{C^{n+1}}{2 !}+\ldots \simeq C^{n+1}-\frac{\Delta t}{6} \frac{\partial C^{n+1}}{\partial t} \\
C^{n+1 / 6}=\frac{C^{n}}{0 !}+\left(\frac{\Delta t}{6}\right) \frac{\partial}{\partial t} \frac{C^{n}}{1 !}+\left(\frac{\Delta t}{6}\right)^{2} \frac{\partial^{2}}{\partial t^{2}} \frac{C^{n}}{2 !}+\ldots \simeq C^{n}+\frac{\Delta t}{6} \frac{\partial C^{n}}{\partial t} .
\end{gathered}
$$

As equações (6) e (7) podem ser utilizadas para reescrever a equação (5) como

$$
C^{n+1}-\frac{1}{2} \Delta t \frac{\partial C^{n+5 / 6}}{\partial t}=C^{n}+\frac{1}{2} \Delta t \frac{\partial C^{n+1 / 6}}{\partial t} .
$$


Novamente utilizando a série de Taylor,

$$
\begin{aligned}
C^{n+1 / 2} & =\frac{C^{n}}{0 !}+\left(\frac{\Delta t}{2}\right) \frac{\partial}{\partial t} \frac{C^{n}}{1 !}+\left(\frac{\Delta t}{2}\right)^{2} \frac{\partial^{2}}{\partial t^{2}} \frac{C^{n}}{2 !}+\ldots \\
& \simeq C^{n}+\frac{\Delta t}{2} \frac{\partial C^{n}}{\partial t} \simeq C^{n}+\frac{\Delta t}{2} \frac{\partial C^{n+1 / 6}}{\partial t} .
\end{aligned}
$$

A partir das equações (8) e (9), tem-se

$$
C^{n+1}-\frac{\Delta t}{2} \frac{\partial C^{n+5 / 6}}{\partial t}=C^{n+1 / 2}
$$

As equações (6), (7), (9) e (10) formam, juntas, o sistema

$$
\left\{\begin{array}{l}
C^{n+1 / 6}=C^{n}+\frac{\Delta t}{6} \frac{\partial C^{n}}{\partial t} \\
C^{n+1 / 2}=C^{n}+\frac{\Delta t}{2} \frac{\partial C^{n+1 / 6}}{\partial t} \\
C^{n+5 / 6}=C^{n+1}-\frac{\Delta t}{6} \frac{\partial C^{n+1}}{\partial t} \\
C^{n+1}=\frac{\Delta t}{2} \frac{\partial C^{n+5 / 6}}{\partial t}+C^{n+1 / 2}
\end{array}\right.
$$

Por meio de algumas substituições, o sistema (11) pode ser simplificado a uma única equação, dada por

$$
C^{n+5 / 6}+\frac{\Delta t}{6} \frac{\partial C^{n+1}}{\partial t}-\frac{\Delta t}{2} \frac{\partial C^{n+5 / 6}}{\partial t}=C^{n+1 / 6}-\frac{\Delta t}{6} \frac{\partial C^{n}}{\partial t}+\frac{\Delta t}{2} \frac{\partial C^{n+1 / 6}}{\partial t} .
$$

\section{Tratamento das derivadas}

No que diz respeito as derivadas presentes na equação (12), as temporais podem ser substituídas pela própria equação (1), ou seja,

$$
\frac{\partial C^{n}}{\partial t}=D_{x x} \frac{\partial^{2} C^{n}}{\partial x^{2}}+D_{y y} \frac{\partial^{2} C^{n}}{\partial y^{2}}-v_{x} \frac{\partial C^{n}}{\partial x}-v_{y} \frac{\partial C^{n}}{\partial y}-k C^{n} .
$$

Note que até o momento estão omitidos os índices espaciais. Para os demais níveis de tempo basta substituir $n$ pelo nível de tempo desejado. Além disso, utilizando diferenças centrais de segunda ordem para as derivadas espaciais de ordem dois e diferenças atrasadas de primeira ordem para as derivadas espaciais de ordem um, tem-se

$$
\begin{array}{r}
C_{i, j}^{n+5 / 6}+\frac{\Delta t}{6}\left[D_{x x} \frac{C_{i+1, j}^{n+1}-2 C_{i, j}^{n+1}+C_{i-1, j}^{n+1}}{(\Delta x)^{2}}+D_{y y} \frac{C_{i, j+1}^{n+1}-2 C_{i, j}^{n+1}+C_{i, j-1}^{n+1}}{(\Delta y)^{2}}\right. \\
\left.-v_{x} \frac{C_{i, j}^{n+1}-C_{i-1, j}^{n+1}}{\Delta x}-v_{y} \frac{C_{i, j}^{n+1}-C_{i, j-1}^{n+1}}{\Delta y}-k C_{i, j}^{n+1}\right]+ \\
+\left[-\frac{\Delta t}{2}\right]\left[D_{x x} \frac{C_{i+1, j}^{n+5 / 6}-2 C_{i, j}^{n+5 / 6}+C_{i-1, j}^{n+5 / 6}}{(\Delta x)^{2}}+D_{y y} \frac{C_{i, j+1}^{n+5 / 6}-2 C_{i, j}^{n+5 / 6}+C_{i, j-1}^{n+5 / 6}}{(\Delta y)^{2}}\right. \\
\left.-v_{x} \frac{C_{i, j}^{n+5 / 6}-C_{i-1, j}^{n+5 / 6}}{\Delta x}-v_{y} \frac{C_{i, j}^{n+5 / 6}-C_{i, j-1}^{n+5 / 6}}{\Delta y}-k C_{i, j}^{n+5 / 6}\right]
\end{array}
$$


(...continuação)

$$
\begin{array}{r}
=C_{i, j}^{n+1 / 6}-\frac{\Delta t}{6}\left[D_{x x} \frac{C_{i+1, j}^{n}-2 C_{i, j}^{n}+C_{i-1, j}^{n}}{(\Delta x)^{2}}+D_{y y} \frac{C_{i, j+1}^{n}-2 C_{i, j}^{n}+C_{i, j-1}^{n}}{(\Delta y)^{2}}\right. \\
\left.-v_{x} \frac{C_{i, j}^{n}-C_{i-1, j}^{n}}{\Delta x}-v_{y} \frac{C_{i, j}^{n}-C_{i, j-1}^{n}}{\Delta y}-k C_{i, j}^{n}\right]+ \\
+\frac{\Delta t}{2}\left[D_{x x} \frac{C_{i+1, j}^{n+1 / 6}-2 C_{i, j}^{n+1 / 6}+C_{i-1, j}^{n+1 / 6}}{(\Delta x)^{2}}+D_{y y} \frac{C_{i, j+1}^{n+1 / 6}-2 C_{i, j}^{n+1 / 6}+C_{i, j-1}^{n+1 / 6}}{(\Delta y)^{2}}\right. \\
\left.-v_{x} \frac{C_{i, j}^{n+1 / 6}-C_{i-1, j}^{n+1 / 6}}{\Delta x}-v_{y} \frac{C_{i, j}^{n+1 / 6}-C_{i, j-1}^{n+1 / 6}}{\Delta y}-k C_{i, j}^{n+1 / 6}\right] .
\end{array}
$$

A equação (14) corresponde à discretização da equação de transporte.

\section{Níveis de tempo intermediários}

Diferente do método de Crank-Nicolson, nos quais os níveis de tempo intermediários desaparecem, esse método os conserva na discretização da equação. Como o problema fornece, em geral, a solução em apenas um dos níveis de tempo, que corresponde à condição inicial, para poder trabalhar a equação (14) é necessário adotar alguma estratégia. Veja algumas:

(A) $C^{n+5 / 6} \simeq C^{n+1}$ e $C^{n+1 / 6} \simeq C^{n}$;

(B) $C^{n+5 / 6} \simeq \frac{5}{6} C^{n+1}+\frac{1}{6} C^{n}$ e $C^{n+1 / 6} \simeq \frac{1}{6} C^{n+1}+\frac{5}{6} C^{n}$;

(C) $C^{n+1 / 6}=C^{n}+\frac{\Delta t}{6} \frac{\partial C^{n}}{\partial t}$ e $C^{n+5 / 6} \simeq \frac{5}{6} C^{n+1}+\frac{1}{6} C^{n}$;

(D) Resolver o sistema (11) sem utilizar a Eq. (14).

Para simplificar a notação, os esquemas obtidos por meio das estratégias (A), (B), (C) e (D) serão referidos apenas como $\mathrm{PADE} / \mathrm{A}, \mathrm{PADE} / \mathrm{B}, \mathrm{PADE} / \mathrm{C}$ e PADE/D, respectivamente.

As estratégias de solução dos esquemas PADE/A, PADE/B e PADE/C são semelhantes, visto que todas envolvem a resolução de um sistema linear. Utilizando como base o método de Gauss-Seidel, isola-se $C_{i, j}^{n+1}$ nas discretizações de cada esquema e faz-se um "chute" inicial para a solução no nível de tempo $n+1$, por exemplo, zero. Para o esquema PADE/C faz-se ainda necessário o cálculo de $C^{n+1 / 6}$, que é realizado de maneira direta a partir da equação (I).

A estratégia empregada no PADE/D se assemelha ao raciocínio feito por [4], em que são discutidos alguns métodos de múltiplo estágio utilizando aproximantes de Padé. Tais métodos são atribuídos Amiram Harten (1946-1994) e Hillel Tal-Ezer (ver [5]).

Basicamente, para esse caso, é necessário resolver dois estágios explícitos, dados pelas equações (I) e (II) do sistema (11) e dois estágios implícitos, dados pelas equações (III) e (IV).

Uma das maneiras de resolver esse problema é a seguinte:

1. de posse das condições iniciais, as equações (I) e (II) são calculadas diretamente, nessa ordem, sendo necessário apenas algumas adequações para os termos de fronteira;

2. aproxima-se a concentração no nível de tempo $n+5 / 6$ por meio de uma extrapolação quadrática utilizando a concentração nos níveis de tempo $n, n+1 / 6$ e $n+1 / 2$;

3. calcula-se a concentração no nível de tempo $n+1$ utilizando a equação (IV);

4. por meio da equação (III) recalcula-se a concentração no nível de tempo $n+5 / 6$;

5. recalcula-se $C^{n+1}$ e $C^{n+5 / 6}$, sucessivamente, até obter convergência para $C^{n+1}$. 


\section{Simulações numéricas}

A fim de verificar a eficácia nas aproximações, foram feitos testes preliminares com a realização de cortes no domínio a fim de transformá-lo em um problema unidimensional, o qual já é bem conhecido na literatura e possui solução analítica (ver [6]).

Considere, para os testes iniciais, $v_{x}=1, D_{x x}=0.2, \Delta x=0.1,0 \leq x \leq 2, k=0.001, v_{y}=0$, $D_{y y}=0.0, \Delta y=1.0,0 \leq y \leq 2$ e $\Delta t=0.002$.

O problema consiste em analisar a dispersão de determinado poluente lançado constantemente na fronteira $x=0$, de modo que $C(0, y, t)=1$ para $t \geq 0$ e $0 \leq y \leq 2$. Como o objetivo é comparar os diferentes esquemas com a solução analítica realiza-se um corte no domínio na direção $x$, por exemplo, em $y=1$, a fim de observar o problema como se fosse unidimensional.

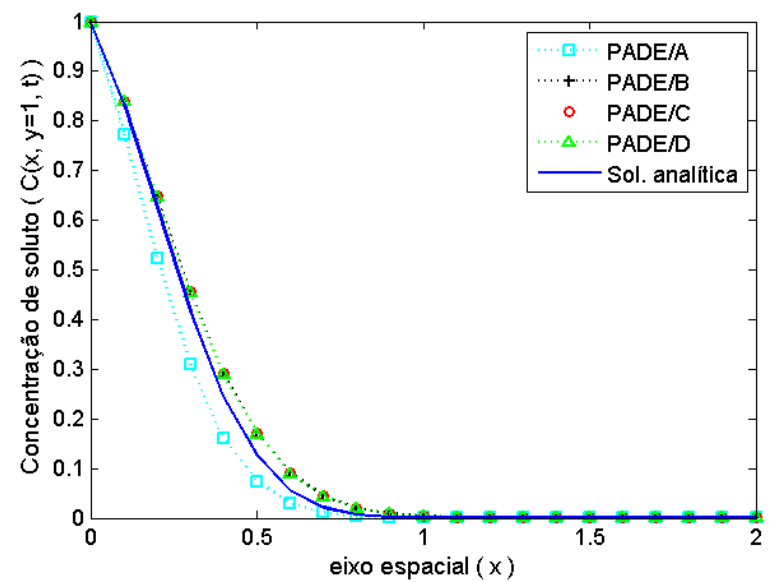

Figura 1: Solução analítica e aproximações utilizando os métodos PADE/A, PADE/B, PADE/C e PADE/D. (simulação 1)

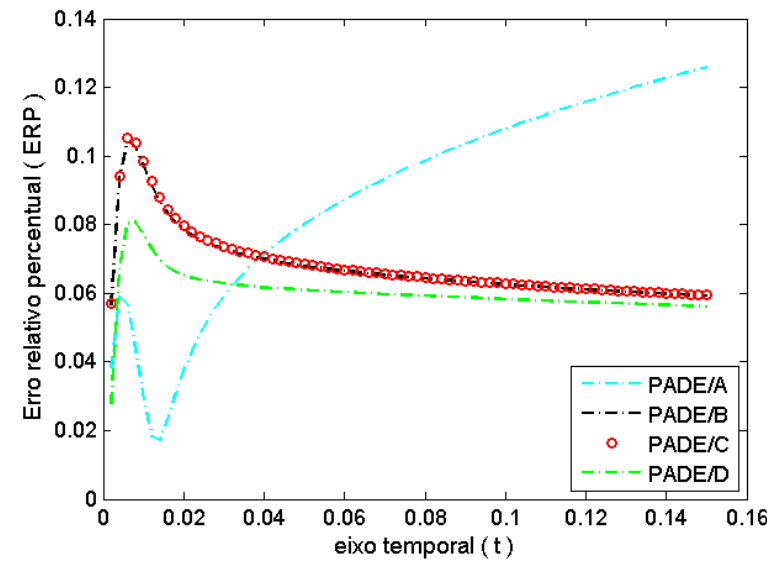

Figura 2: Erro relativo percentual (ERP) em cada nível de tempo. Para esta simulação (simulação 1) foi utilizado $\Delta t=0.002, D_{x x}=0.2$ e $\Delta x=0.1$, resultando em $P e_{\text {grid }}=0.5$.

Note que o método PADE/A resultou em maiores erros. Isso se repete em outras simulações utilizando outros parâmetros. O valor adimensional $P e_{\text {grid }}$ é conhecido como número de Péclet, dado por $P e_{\text {grid }}=v_{x} \Delta x / D_{x x}$. Referente aos resultados apresentados nas Figuras 1 e 2, tem-se:

\begin{tabular}{|c|c|c|c|c|}
\hline Método & PADE/A & PADE/B & PADE/C & PADE/D \\
\hline Tempo (em segundos, da simulação 1) & 14.5 & 14.9 & 26.7 & 18.5 \\
\hline
\end{tabular}

As simulações podem ser refeitas com algumas modificações nos parâmetros.

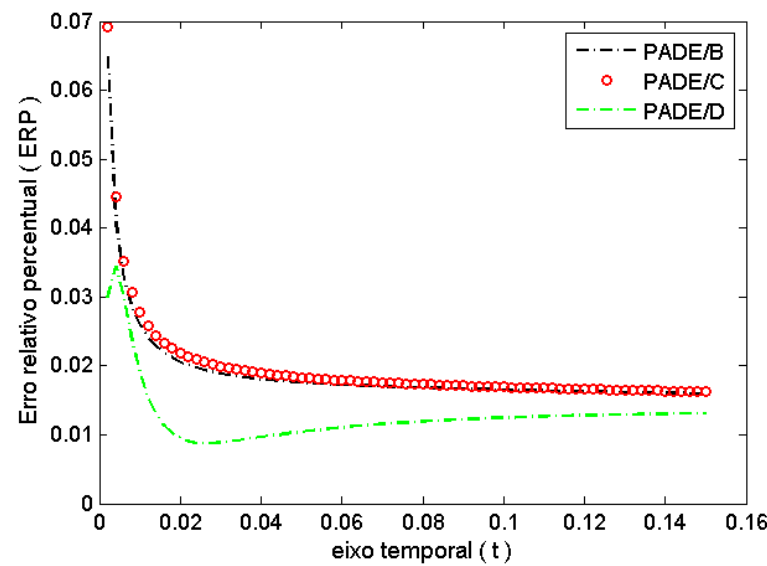

Figura 3: Erro relativo percentual (ERP) em cada nível de tempo. Para esta simulação (simulação 2) foi utilizado $\Delta t=0.002, D_{x x}=1.0$ e $\Delta x=0.1$, resultando em $P e_{\text {grid }}=0.1$.

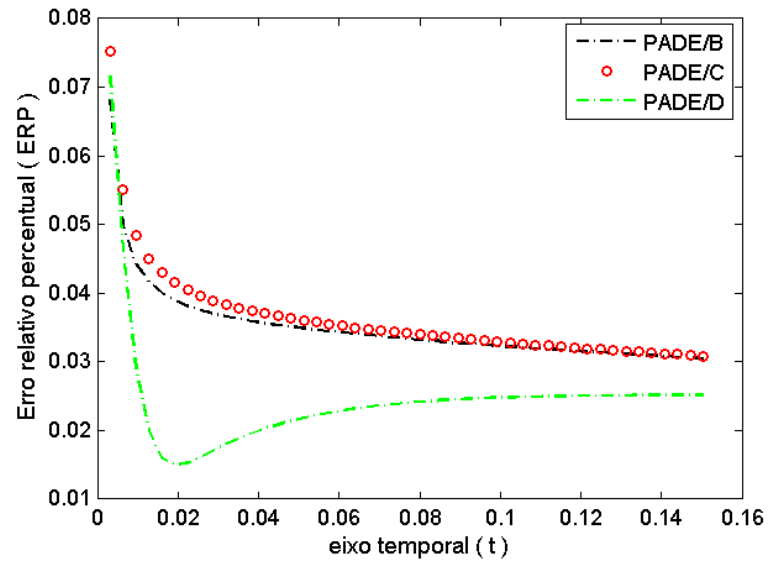

Figura 4: Erro relativo percentual (ERP) em cada nível de tempo. Para esta simulação (simulação 3) foi utilizado $\Delta t=\Delta x / 15, D_{x x}=0.2$ e $\Delta x=0.048$, resultando em $P e_{\text {grid }}=0.24$. 
Novamente em relação ao tempo computacional, tem-se:

\begin{tabular}{|c|c|c|c|}
\hline Método & PADE/B & PADE/C & PADE/D \\
\hline Tempo (em segundos, da simulação 2) & 1.7 & 2.1 & 1.2 \\
\hline Tempo (em segundos, da simulação 3) & 1.9 & 3.2 & 2.3 \\
\hline
\end{tabular}

\section{Conclusão}

O objetivo deste trabalho foi aplicar um método de múltiplo estágio (conhecido como método de Harten/Tal-Ezer) ao estudo da equação de transporte bidimensional (2D). Dentre as estratégias utilizadas destacam-se a (B) e a (D), geradoras dos métodos PADE/B e PADE/D. Foi observado que a utilização do $\mathrm{PADE} / \mathrm{B}$ conduz às mesmas equações que poderiam ser obtidas utilizando o método de Crank-Nicolson, já bastante difundido na literatura. Com base nestes testes preliminares nota-se que o método PADE/D, o qual segue o raciocínio utilizado por [4], gera melhores aproximações que o método de Crank-Nicolson. Além disso, é perceptível que a eficácia do método PADE/A na aproximação da solução é menor que os demais métodos discutidos. Em relação ao tempo computacional, percebe-se que o método PADE/C é mais lento, desistimulando sua utilização. Quando comparadas as estratégias (B) e (D), o tempo computacional é relativamente semelhante e dependente dos parâmetros fornecidos. Isso ocorre devido à velocidade de convergência de cada método. Entretanto, como foi dito, as simulações encontram-se em fase preliminar. O tempo computacional e o número de iterações utilizado ainda é muito pequeno para se tirar conclusões acerca do desempenho. Objetivos a curto prazo para o avanço deste trabalho são a realização de mais simulações explorando a variação dos parâmetros. Espera-se determinar regiões de estabilidade e de melhor desempenho para cada método.

\section{Referências}

[1] M.C.K. Aguilera-Navarro et al., Os aproximantes de Padé, Matemática Universitária 26 (1999) 27.

[2] B. Ataie-Ashtiani \& S.A. Hosseini, Numerical errors of explicit finite difference approximation for two-dimensional solute transport equation with linear sorption, Environmental Modelling and Software, 20 (2005) 817-826.

[3] E. Corcoran et al., Sick water, The central role of wastewater management in sustainable development. A rapid response assessment, United Nations Environment Programme. UNHABITAT, GRID. Arendal, (2010).

[4] J. Donea, B. Roig \& A. Huerta, High-order accurate time-stepping schemes for convectiondiffusion problems, Computer methods in applied mechanics and engineering, 182 (2000) 249-275.

[5] A. Harten \& H. Tal-Ezer. On a fourth order accurate implicit finite difference scheme for hyperbolic conservation laws. I. Nonstiff strongly dynamic problems, Mathematics of Computation, 36 (1981) 353-373.

[6] M.Th. Van Genuchten, Analytical solutions for chemical transport with simultaneous adsorption, zero-order production and first-order decay, Journal of Hydrology, 49 (1981) 213-233. 